\title{
Retention functions for syntactic and lexical vs semantic information in sentence recognition memory
}

\author{
IAN BEGG \\ McMaster University, Hamilton, Ontario, Canada \\ and \\ WAYNE A. WICKELGREN \\ University of Oregon, Eugene, Oregon 97403
}

\begin{abstract}
In a continuous recognition memory design, Ss judged whether each sentence was identical in form and meaning to some previously presented sentence, then judged whether the sentence was identical in meaning irrespective of form, and, finally, rated the likelihood of recognizing the sentence if it was presented an hour later (memorability). The Ss were given sentences that were new, identical to, or paraphrased from some previously presented sentence, at delays ranging from $0 \mathrm{sec}$ to $2 \mathrm{~h}$. Long-term memory for both semantic information and syntactic-lexical information decayed according to the same exponential-power retention function previously found to be characteristic of the decay of simpler verbal materials (nonsense items, letters, digits, words, and word pairs). Semantic memory primarily differed from syntactic-lexical memory in that the semantic information had a far higher degree of learning, but the decay rate for syntactic-lexical information was also approximately $50 \%$ greater than the decay rate for semantic information.
\end{abstract}

The present paper represents a convergence of two interests. First, we wish to extend the study of the basic dynamics of long-term retention from simple verbal materials (e.g., Wickelgren, 1972a) to more complex verbal materials, as represented by sentences. Second, we wish to compare long-term retention for semantic information with that for syntactic and lexical information.

Wickelgren (1972b) demonstrated that a single type of long-term memory trace with an "exponential-power" retention function accounts for long-term retention functions over the range from tens of seconds to over 2 years for a wide variety of simple verbal materials (nonsense materials, words, and word pairs) under a wide variety of conditions and rates of presentation. The best fitting exponential-power retention function for the long-term trace was as follows:

$$
d_{a}=\lambda e^{-\psi} t^{1-\gamma}
$$

where $d_{a}$ represents the strength of the long-term trace, $\lambda$ is the degree of learning in long-term memory, $\psi$ is the decay rate, and $\gamma$ is the exponent of growth of resistance of the long-term trace $(\gamma=.75)$. Thus, for simple verbal materials, $\log$ strength $\left(\log d_{a}\right)$ decreases linearly as a

*This research was begun jointly at M.I.T. and continued separately at the University of Western Ontario, McMaster University, and the University of Oregon. The work was supported by Grants MH 08890 and MH 17958 from NIMH and Grant 3-0097 from the National Institu te of Education to Wayne Wickelgren, by Grant NsG 496 from NASA to Hans-Lukas Teuber, by Contract F44620-73-C-0056 from ARPA to Ray Hyman, by an NSF grant to the Mathematical Social Science Board to support a summer conference on Mathematical Models of Memory held at M.I.T. in July 1969, by a National Research Council of Canada postgraduate scholarship and by National Research Council of Canada Grant A8122 to Ian Begg. function of delay time raised to the .25 power, with an initial intercept (at zero retention interval) equal to $\log \lambda$ and a slope parameter equal to the decay rate $(\psi)$.

Wickelgren (1972a) rejected a variety of alternative forms for the retention function, including linear decay $\left(d_{a}=\lambda-\psi t\right)$, exponential decay $\left(d_{a}=\lambda e^{-\psi t}\right)$, $\operatorname{logarithmic}$ decay $\left(\mathrm{d}_{\mathrm{a}}=\lambda-\psi \log \mathrm{t}\right)$, and power-function decay $\left(d_{a}=\lambda t-\psi\right)$. In addition, Wickelgren (1972b) determined that the optimal exponent for the growth of trace resistance was in the vicinity of $\gamma=.75$. Wickelgren (1972a) obtained the exponential-power form of the retention function for individual Ss without distortion due to pooling or other unjustified averaging over individual Ss. Finally, Wickelgren (1972a) demonstrated that the exponential-power form of the long-term retention function did not result from averaging over items with different degrees of rated memorability, since the same form was obtained for subsets of items given different degrees of rated memorability by Ss, although the decay rate was slightly lower for items with highly rated memorability.

The present study attempts to determine whether the same exponential-power retention function fits the retention of semantic and syntactic-lexical information in recognition memory for sentences. A variety of studies have indicated that semantic information is better retained than syntactic and lexical information in long-term memory. Sachs $(1967 \mathrm{a}, \mathrm{b})$ demonstrated that changes in the meaning of sentences were more often correctly reported than were changes in the lexical and syntactic properties of sentences. Sachs's sentences were presented in connected discourse and tested after different amounts of interpolated material from the passage. Although Sachs (1967a) reported that "recognition memory for the form of the sentence 
declines much more rapidly than recognition memory for the meaning," it is not possible to determine the decay rate of long-term memory for semantic vs syntactic and lexical (formal) changes, since the maximum retention interval used by Sachs was approximately $46 \mathrm{sec}$ (containing 160 syllables). It is well established (e.g., Shepard, 1967; Wickelgren, 1972a) that long-term memory for verbal materials of all kinds declines very little over such a short period of time. In fact, if the 0 -sec retention interval is excluded on the grounds that it contains an undetermined amount of short-term memory, the remaining portions of the retention functions in Sachs $(1967 \mathrm{a}, \mathrm{b})$ demonstrate little forgetting over this short retention interval, in congruence with all previous studies.

The primacy of semantic memory over syntactic-lexical memory was demonstrated in an entirely different manner by Bransford and Franks (1971) and Franks and Bransford (1972), who demonstrated that Ss combine the information expressed in separate but semantically related sentences into "wholistic" semantic ideas. These Ss demonstrated high false recognition rates for new sentences that incorporated all of the information characteristic of the complete idea. Other studies have distinguished between semantic memory and memory for deep structure, indicating that it is primarily the semantic information that is retained rather than the deep structure information (Bransford, Barclay, \& Franks, 1972; Johnson-Laird \& Stevenson, 1970). The present paper is not concerned with making distinctions between deep structure, surface structure, and lexical information. Rather, all of this information will be lumped together under the rubric "syntactic-lexical" information, and the retention of this will be contrasted with the retention of semantic information.

Using a continuous recognition memory design with independent concrete sentences (not connected discourse), Begg (1971) also demonstrated that semantic changes were more accurately identified than syntactic and lexical changes at delays ranging from $10 \mathrm{sec}$ to $5 \frac{1}{2} \mathrm{~min}$ for visual presentation and at delays ranging from $20 \mathrm{sec}$ to $11 \mathrm{~min}$ for auditory presentation. The delays used by Begg probably excluded any substantial contribution from short-term memory and, thus, reflect only long-term memory. Begg found that, while semantic memory declined with increasing retention interval, syntactic and lexical memory declined little, if at all, with increasing retention interval. Although Begg employed a much larger range of appropriate long-term retention intervals than did Sachs, the range employed by Begg is still far too small to demonstrate a substantial loss in long-term retention. Thus, a comparison of the decay rates over such short time intervals will largely reflect chance fluctuation.

Despite the fact that semantic memory appears to be much more accurate than syntactic-lexical memory and that the experiments by Sachs $(1967 \mathrm{a}, \mathrm{b})$ and Begg
(1971) showed little evidence of syntactic-lexical long-term decay, the syntactic-lexical judgments were above a chance level in accuracy in the above mentioned experiments, as they were in another experiment by Begg and Paivio (1969). Consequently, some syntactic-lexical information appears to be represented in memory even after $11 \mathrm{~min}$. Thus, the second major purpose of the present study was to determine whether there are any differences in the form and rate of the retention function for semantic information, on the one hand, and syntactic and lexical information, on the other hand, in recognition memory for sentences, employing a much larger range of retention intervals.

\section{METHOD}

Materials

\section{Sentence Selection}

Thirty-six hundred sentences, all of which had occurred previously in print, were selected for the present study. A few were taken from previous studies, but the majority were selected from 47 books, chosen semirandomly from the junior high section of the Boston Public Library. Twenty of the books were of the adventure variety (six at sea, six in the days of knights, two in war, four in modern settings, and two quasihistories). There were five Westerns, two animal stories, three science fictions, and four mysteries. There were five historical novels (one on the French Revolution, one about King Richard, one on Vikings, one on the early Japanese settlements in California, and one on the climbing of the Matterhorn), three sports stories (basketball, football, and ancient Graecian Olympics), and, finally, two biographies (Annie Sullivan and Rudyard Kipling).

The first complete paragraph on the fourth page of each book was used to select sentences. Sentences were excluded if they contained or were part of direct discourse or if they contained proper nouns. Only sentences between 6 and 20 syllables were chosen. An attempt was made to use only sentences that seemed quite concrete to the selector. In this fashion, 3600 sentences were selected.

Paraphrase Construction. At the next stage, each of the 3600 sentences was rewritten in such a way that its meaning was preserved, but the form in which the meaning was expressed was altered. The types of changes made are outlined below.

(1) Changes in Order. The order of the words in the sentence was changed, but no words were added or deleted. e.g. He ran quickly down the street.

He ran down the street quickly. e.g. The tall lamp was very bright.

The very bright lamp was tall.

(2) Formal Syntactic Changes. (a) Addition or deletion of unimportant features and (b) tense changes. e.g. ... he wanted to have freedom.

... he wanted freedom. e.g. While he worked ...

While he was working ...

(3) Voice Changes. Active sentences were restated in passive voice and vice versa.

e.g. The boy was taken to the hospital by his parents.

The parents took their boy to the hospital.

(The "his" to "their" change is not exactly an active to passive change, but it does preserve meaning.)

(4) Synonym Changes. This is a broad heading including all unit replacements, such as a clause for an adjective, noun for a noun, etc. e.g. . . . the red-headed boy ...

... the boy who had red hair ... 
(5) Probabilistic Extensions or Deletions. This category includes the addition or deletion of components of the sentences which, given the rest, are so likely that they are redundant.

e.g. He cut himself while he was shaving.

He cut himself with his razor while he was shaving.

e.g. The boy with red hair ...

The boy with red hair on his head...

e.g. He held a pencil ... .

He held a pencil in his hand ...

(6) Directional Emphasis Changes. This category includes changes that involve positive to negative changes. e.g. Most of the ...

All but a few of the... e.g. Almost all did ...

Only a few did not...

(7) Combinations of the Above.

The changes were made with roughly equivalent frequencies but no count was made.

\section{Procedure}

Each $S$ served for $3 \frac{1}{2} \mathrm{~h}$ on 4 consecutive days. Sentences were presented visually with 25 on each page. The S slid a cardboard rider along the page so that each sentence appeared in the slot for $7.5 \mathrm{sec}$, or a rate of eight sentences per minute. An electronic timer produced a tone every $7.5 \mathrm{sec}$ to pace the presentation.

As each sentence was exposed, the $S$ read it and made three responses. First, he responded "same" or "different," with confidence from 1 (low) to 4 (high), if he thought the sentence was identical in form and meaning to one with which he had been previously presented (structure judgment). Second, he responded "same" or "different," with confidence from 1 to 4 , if he thought the meaning of the sentence was identical to a sentence presented earlier in the session (meaning judgment). If the first response was "same," so was the second. If the first response was "different," the second could be "same" if the S recognized the meaning but not the form or "different" if he recognized neither meaning nor form, as if the item were new. The third response was an estimate of the probability with which the $S$ thought he could recognize the meaning of the sentence if it were presented again after $1 \mathrm{~h}$ (memorability).

\section{Design}

There were 31 conditions in the experiment. One condition, the new condition, was the first presentation of any sentence. There were 15 same conditions in which the sentence was identical in form and meaning to one that had been presented previously and 15 paraphrase conditions in which the (syntactic or lexical) form of the sentence differed from the form in which it was first presented, but the meaning was preserved. The 15 conditions within each of the same and paraphrase conditions were different delays between the first presentation of the sentence and the second presentation. In terms of intervening sentences, the delays were $0,1,2,3,4,5,6,8,16,32,48,96$, 200,480 , and 960 sentences corresponding to $0,7.5,15,22.5$, $30,37.5$, and $45 \mathrm{sec}, 1,2,4,6,12,25,60$, and $120 \mathrm{~min}$.

The $3 \frac{1}{2}-\mathrm{h}$ session was split into a pre half hour, a first, second, and third hour. Responses made during the pre half hour were not scored. Of the 280 items presented in the pre half hour, 144 were news, 48 were sames ( 8 each of $0,1,2,5,16$, and 48 intervening items), and 48 were paraphrases. Of the 480 items in the first hour, 256 were news, 112 were sames ( 8 each of $0-480$ intervening items; the 480 condition could only be represented in the last half of the hour), and 112 were paraphrases. In the second hour, 240 items were news, 120 were sames ( 8 each of all delays; the 960 only in the last half), and 120 were paraphrases. In the third hour, 224 were news, 128 were sames ( 8 of all conditions, with an additional 8 of 960), and 128 were paraphrases. Thus, for the $3 \mathrm{~h}$ that were scored, an average of
240 new items per hour and an average of $8 / \mathrm{h}$ of each of the 15 sames and 15 paraphrase conditions were presented. The sentences were assigned randomly to conditions. In addition, either the original sentence or the paraphrase of it served as the new sentence, again determined at random.

In the 4-day period, any $S$ saw 6720 sentences, about half of which were different. He made 5760 scored responses, 1440 per day. Sentences were not repeated from 1 day to the next. Over the 4 days, there were thus 96 paraphrases and sames at each delay interval and 2880 news.

\section{Subjects}

The Ss were seven paid volunteers recruited through the M.I.T. student employment office.

\section{RESULTS AND DISCUSSION}

First, to determine whether the procedure of having structure judgments always precede meaning judgment had some large biasing effect on the responses, the overall proportion of "yes" and "no" judgments was calculated. The structure judgment was "yes" in .241 of the cases (.25 of the cases required "yes" responses). The second response was "yes" in .512 of the cases $(.5$ of the cases required "yes" responses). Thus, the procedure of having structure judgments precede meaning judgments did not result in much deviation in the proportion of "yes" responses from the actual distribution of sentence types.

Using the methods of statistical decision theory as described in Wickelgren (1972a), memory strength discriminability values ( $d_{a}$ values) were obtained from memory operating characteristics. The $d_{a}$ value is simply a lower variance estimate of $\mathrm{d}^{\prime}$ and represents the difference in mean strengths of two conditions (such as same vs new). The unit of measurement is the standard deviation of the strength distribution for some fixed condition (such as new). Three types of memory operating characteristics were plotted. First and second, recognition memory for semantic information was assessed by plotting the rating probabilities for the meaning judgments for the same conditions at each delay against the new condition and also by plotting the rating probabilities for the paraphrase condition at each delay against the new condition. Third, recognition memory for syntactic-lexical information was assessed by plotting the rating frequencies for the structure judgments for the same vs paraphrase condition at each delay. Note that in the third comparison the meanings of the test sentences in the same and paraphrase conditions are identical (or close to identical) to the original condition, and it is primarily the syntactic and lexical structure that differentiates the two classes of sentences. In the first two comparisons, it is only the semantic equivalence of the two sentences that Ss are supposed to judge and, therefore, such comparisons should depend largely or entirely on memory for semantic information. Obtaining equivalent results for the two semantic comparisons (same vs new and paraphrase vs new) would indicate that there was no interaction between semantic 


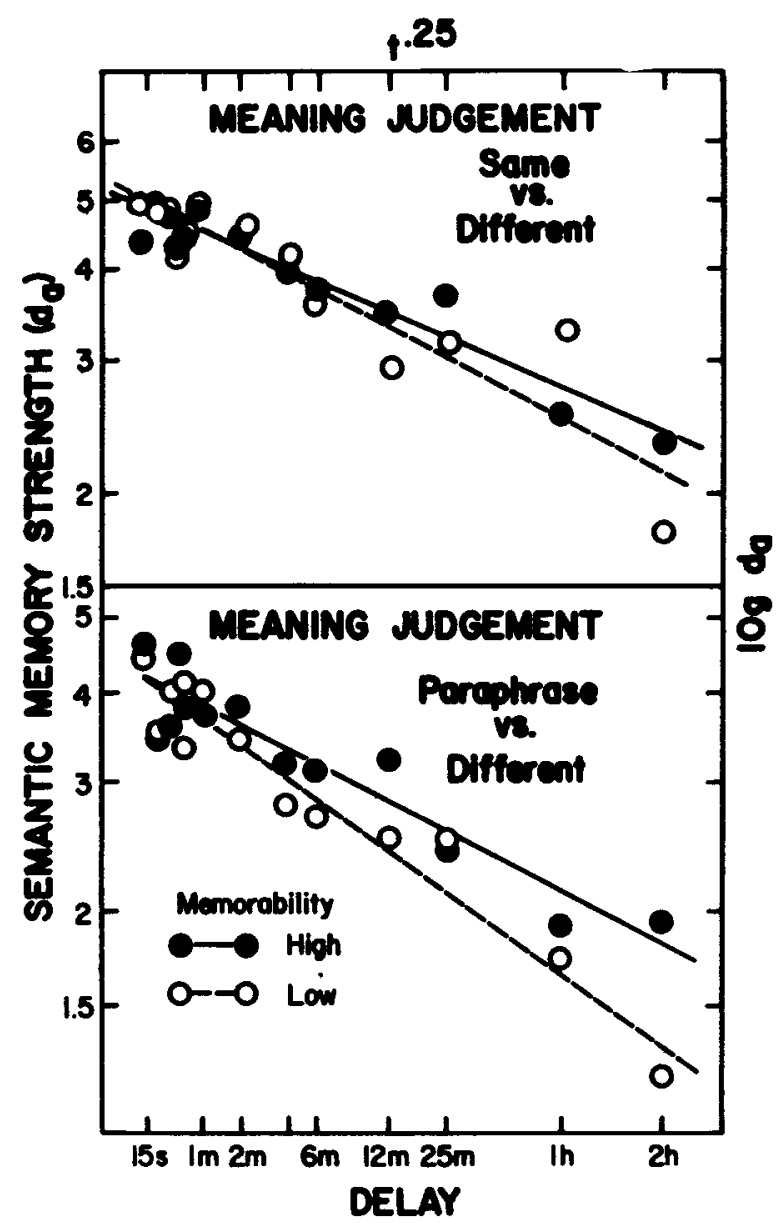

Fig. 1. Semantic strength retention functions for same vs different and paraphrases vs different sentences with both highand low-memorability ratings (meaning judgments).

and syntactic-lexical information in retrieval from semantic memory and that the paraphrases were perfect in preserving meaning. Any tendency for the paraphrases to be less discriminable from new sentences than from same sentences would indicate either an interaction between semantic and syntactic-lexical memory or else that the paraphrases did not perfectly preserve meaning.

\section{Form of the Retention Function}

Each of the three previously specified retention functions was studied separately for sentences rated low or high in memorability (rated likelihood of recognizing the sentence after an hour). The shortest two retention intervals, 0 and $7.5 \mathrm{sec}$, were deleted from the retention functions to eliminate any possible contamination from short-term memory. The resulting six strength retention functions are shown in Figs. 1 and 2.

Strength retention functions for both semantic and syntactic-lexical sentence recognition memory were best fit by an exponential-power retention function identical in form to that which provides the best fit to long-term recognition memory for simpler verbal materials (e.g., words, word pairs, Russian-English word pairs, and letters and digits) as found by Wickelgren (1972b). The basic strength retention function is of the form

$$
\mathrm{d}_{\mathrm{a}}=\lambda \mathrm{e}-\psi \mathrm{t} \cdot 25
$$

where the exponent of $t$ is the same as that found to provide the best fit for long-term retention of simpler verbal material. Determining that the optimum exponent was approximately .25 was achieved by investigating the goodness of fit for a variety of exponents ranging from .1 to .7. Although an exponent of .25 was optimal, nearly optimum fits were obtained with exponents ranging anywhere from .15 to .4 . Furthermore, note that the same form of retention function with the same exponent provides the optimal fit for both semantic and syntactic-lexical memory.

Tables 1 and 2 provide least-squares estimates of the degree of learning $(\lambda)$ and decay rate $(\psi)$ parameters in the best fitting exponential-power functions for all the retention functions shown in Figs. 1 and 2 . The absolute goodness of fit is assessed by the $\mathrm{r}^{2}$ value shown in Tables 1 and 2 for each $S$ and for the average over all seven Ss. The average retention function was obtained by averaging the logs of the $d_{a}$ values for each $S$, because this method of averaging will not distort the form of the retention function, according to the theory.

Although there are occasionally some substantial deviations of the empirical $d_{a}$ values from the theoretical function for individual Ss, the deviations are not at all systematic, as shown by the fact that the $\mathrm{r}^{2}$ value for the average is very high and substantially above the average $\mathrm{r}^{2}$ value for the individual $\mathrm{Ss}$ in all cases. (The deviation of the $r^{2}$ for the average $S$ from the average of the $r^{2}$ for individual Ss constitutes a measure of the degree to

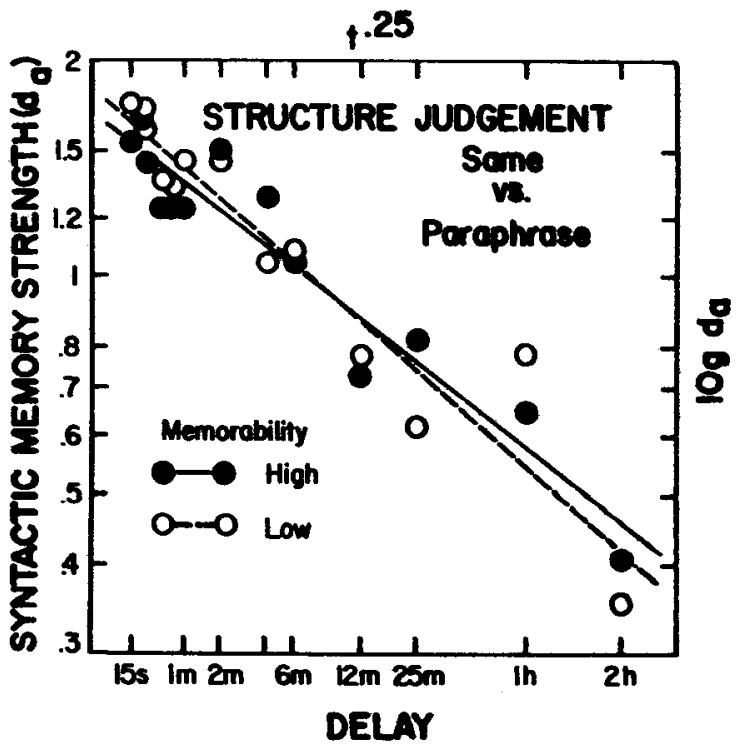

Fig. 2. Syntactic strength retention functions for same vs paraphrase sentences with both high- and low-memorability ratings (structure judgments). 
Table 1

Semantic Recognition Memory for Sentences $\left(d_{a}=\lambda e^{-\psi t} \cdot 25\right)$

\begin{tabular}{|c|c|c|c|c|c|c|c|c|c|c|c|c|}
\hline \multirow[b]{3}{*}{$\mathrm{S}$} & \multicolumn{6}{|c|}{ A. Same vs Different } & \multicolumn{6}{|c|}{ B. Paraphrase vs Different } \\
\hline & \multicolumn{3}{|c|}{ Low Memorability } & \multicolumn{3}{|c|}{ High Memorability } & \multicolumn{3}{|c|}{ Low Memorability } & \multicolumn{3}{|c|}{ High Memorability } \\
\hline & $\lambda$ & $\psi$ & $\mathrm{I}^{2}$ & $\lambda$ & $\psi$ & $r^{2}$ & $\lambda$ & $\psi$ & $r^{2}$ & $\lambda$ & $\psi$ & $r^{2}$ \\
\hline KK & 8.4 & .15 & .70 & 6.3 & .05 & .37 & 6.3 & .15 & .91 & 5.4 & .05 & .39 \\
\hline PR & 3.2 & .10 & .93 & 2.9 & .03 & .20 & 2.8 & .13 & .88 & 3.1 & .06 & .43 \\
\hline $\mathrm{FP}$ & 10.4 & .23 & .81 & 10.6 & .19 & .71 & 7.7 & .30 & .81 & 9.4 & .27 & .78 \\
\hline PF & 7.4 & .10 & .66 & 7.1 & .09 & .54 & 7.2 & .14 & .83 & 5.6 & .07 & .58 \\
\hline $\mathrm{LC}$ & 5.0 & .05 & .45 & 5.0 & .05 & .53 & 4.5 & .11 & .66 & 4.6 & .05 & .56 \\
\hline JT & 5.4 & .07 & .29 & 5.4 & .12 & .78 & 5.9 & .12 & .57 & 4.7 & .15 & .80 \\
\hline MB & 6.9 & .11 & .75 & 6.6 & .15 & .91 & 7.0 & .15 & .91 & 5.5 & .14 & .91 \\
\hline Average & 6.3 & .12 & .85 & 5.9 & .10 & .92 & 5.6 & .16 & .94 & 5.2 & .11 & .89 \\
\hline
\end{tabular}

Note $-\lambda=$ degree of learning, $\psi=$ rate of decay, $r^{2}=$ proportion of variance accounted for by theory.

which the variations for each individual are not systematic across all Ss.) The exponential-power decay function fits retention functions for all conditions extremely well, with a fit at least as good as the fit provided by the same function for the less complex verbal materials in Wickelgren (1972a). At the same time, the empirical retention function departs systematically from linear decay, exponential decay, and power function decay, just as in the previous study of simpler verbal material.

\section{Memorability Ratings}

On the basis of each S's estimate of the probability with which he thought he could later recognize the meaning of the sentence, thie data were subdivided into higher and lower degrees of memorability. As in the Wickelgren (1972a) study, the subdivision did not change the basic exponential-power form of the retention function and had only a modest effect on the decay rate parameters. As in the previous study, high-memorability material had a slightly lower decay rate than low-memorability material. The effect was significant for the semantic memory judgment by a $t$ test on the slope coefficients for the regression lines $(p<.05)$ and the effect was in the same direction, but

Table 2

Syntactic Recognition Memory for Sentences $\left(d_{a}=\lambda e^{-\psi t \cdot 25}\right)$

Same vs Paraphrase

\begin{tabular}{llllllll} 
& \multicolumn{3}{c}{ Low Memorability } & & \multicolumn{3}{c}{ High Memorability } \\
\cline { 2 - 4 } $\mathrm{S}$ & $\lambda$ & $\psi$ & $\mathrm{r}^{2}$ & & $\lambda$ & $\psi$ & $\mathrm{r}^{2}$ \\
\hline KK & 2.8 & .22 & .84 & & 2.5 & .17 & .66 \\
PR & 2.2 & .16 & .80 & & 1.2 & .10 & .18 \\
FP & 2.5 & .19 & .80 & 3.3 & .24 & .64 \\
PF & 2.6 & .18 & .72 & 2.0 & .09 & .34 \\
LC & 1.7 & .11 & .20 & 2.4 & .19 & .80 \\
JT & 1.6 & .13 & .63 & & 1.3 & .09 & .49 \\
MB & 3.5 & .25 & .91 & 3.3 & .27 & .95 \\
Average & 2.4 & .19 & .92 & 2.1 & .17 & .91 \\
\hline
\end{tabular}

Note $-\lambda=$ degree of learning, $\psi=$ rate of decay, $r^{2}=$ proportion of variance accounted for by theory. insignificant, for syntactic-lexical memory. Degree of learning was not different for low vs high rated memorability and was slightly in the direction of a higher degree of learning for material rated less memorable. Thus, as in the previous study, the principal difference between material rated low in memorability and material rated high in memorability was in decay rate, and even these differences were very slight. This may indicate that there truly is little difference in the decay rate parameters for different material or that Ss cannot rate accurately the differences in memorability of different material. However, even when materials of known memorability differences, such as concrete and abstract nouns, are learned to common criterion levels, there is still an advantage in long-term recall of the concrete material over intervals from 2 days to about 1 week (Begg \& Robertson, in press). Thus, the materials do appear to have differential forgetting rates, although it is not possible to assess the sizes of the different rates from the recall data. In any event, in the present experiment there is no indication from this partitioning by categories of rated memorability that the exponential-power form of the retention function results from averaging underlying functions for different classes of items of basically different form. However, the power of this test is considerably less than it was in Wickelgren's (1972a) analysis of cases where substantial differences in the appropriate direction were obtained in degree of learning for material with different degrees of rated memorability.

\section{Semantic vs Syntactic-Lexical Memory}

The results of the present study indicate that long-term memory for both semantic and syntactic-lexical properties of sentences appear to have the same dynamics (same form of retention function), which, in turn, are the same as the dynamics exhibited by long-term memory for word pairs, words, and nonsense verbal material. Thus, there is reason to hope that the same dynamic laws of forgetting will apply for all long-term verbal memory. Note that using a much 
Table 3

Probability of a "Yes" Response

\begin{tabular}{cccccc}
\hline \multirow{2}{*}{$\begin{array}{l}\text { Number of } \\
\text { Intervening }\end{array}$} & \multicolumn{2}{c}{ Meaning Judgment } & & \multicolumn{2}{c}{ Structure Judgment } \\
\cline { 2 - 3 } \cline { 5 - 6 } Sentences & $\begin{array}{c}\text { Same } \\
\text { Sentence }\end{array}$ & $\begin{array}{c}\text { Para- } \\
\text { phrase }\end{array}$ & & $\begin{array}{c}\text { Same } \\
\text { Sentence }\end{array}$ & $\begin{array}{c}\text { Para- } \\
\text { phrase }\end{array}$ \\
\hline 0 & .97 & .95 & .90 & .08 \\
1 & .98 & .94 & .84 & .21 \\
2 & .98 & .96 & .82 & .17 \\
3 & .99 & .94 & .81 & .20 \\
4 & .98 & .93 & .79 & .16 \\
5 & .96 & .96 & .72 & .20 \\
6 & .97 & .93 & .77 & .20 \\
8 & .98 & .94 & .77 & .19 \\
16 & .96 & .92 & .76 & .20 \\
32 & .94 & .89 & .70 & .25 \\
48 & .93 & .88 & .68 & .26 \\
96 & .90 & .86 & .60 & .28 \\
200 & .87 & .79 & .56 & .27 \\
480 & .79 & .66 & .46 & .20 \\
960 & .69 & .65 & .40 & .28 \\
\hline
\end{tabular}

Note-The proportions are the sum of all "yes" responses over the four confidence levels. The probability of a "yes" meaning judgment to a new sentence was .122 and for a "yes" wording judgment was .024. Fifty percent of the sentences had old meanings, while $25 \%$ had old structures. "Yes" structure judg. ments to paraphrases constitute syntactic false alarms.

wider range of retention intervals in the present study demonstrated a substantial decay for both the semantic information, on the one hand, and syntactic-lexical information, on the other hand, in contrast to previous studies that employed smaller ranges of retention intervals, such as Begg (1971), Sachs (1967a,b), and Shepard (1967). These results make the relatively obvious point that it is foolish to attempt to determine forgetting rates over ranges of retention intervals that are small in relation to the rate of forgetting. If an hour is required before substantial changes are observed in long-term retention, one must study retention over an hour. In such cases, decay rates determined over retention intervals of $10 \mathrm{~min}$ or less are very small relative to errors of measurement.

In agreement with the findings of previous studies, memory for the semantic properties of sentences was far greater than memory for the syntactic and lexical properties of sentences. That is to say, the $d_{a}$ values are substantially higher for the semantic retention functions in Fig. 1 than for the syntactic-lexical retention functions in Fig. 2. However, it is clear that the main difference between long-term retention of semantic and syntactic-lexical properties is that the semantic properties have a higher degree of learning to begin with. If Ss normally attend more closely to the meaning of a sentence than to its wording, as Wanner (1968) suggests, different degrees of learning for the meaning and wording are not surprising. Despite different degrees of learning, there is no difference in the basic form of the retention functions for the two types of information and only a modest difference in decay rate.
The proportions of "yes" responses, summed over confidence levels, are presented in Table 3 to provide comparisons with other recognition data. Note that it is not possible to make direct comparisons between meaning and structure judgments because of different false alarm rates and different distributions of "yes" and "no" responses required for the two types of judgment.

Note that the differences in meaning between the same or paraphrase sentences and the new sentences could either be greater or less than the differences in meaning studied by Sachs $(1967 \mathrm{a}, \mathrm{b})$. The alterations used by Sachs were systematic changes in originally presented sentences (switching subjects and objects, negation of a sentence, or substitution of a word occurring elsewhere in the passage). While the difference in meaning between two completely unrelated sentences may seem to be greater than the difference in meaning between a sentence and a systematically changed version of the same sentence, to our knowledge no study has ever contrasted the discriminability of the two types of comparison.

From Tables 1 and 2, it is apparent that the degree of learning parameter $(\lambda)$ is approximately $2 \frac{1}{2}$ times greater for semantic information than for the syntactic-lexical information in this experiment, while the decay rate for syntactic-lexical information is only about $50 \%$ greater than the decay rate for semantic information. There obviously exists a long-term memory for syntactic and lexical information, though the degree of learning is substantially lower than for semantic information. The decay rate for syntactic-lexical information is somewhat higher too, but it is still of the same order of magnitude as the decay rate for semantic information, under the conditions of this experiment. Note that the differences in apparent decay rate might be substantially greater in connected discourse, where the semantic information probably receives repetition from subsequent context, while the syntactic information does not. The beneficial effects of contextual repetition in discourse were recently demonstrated by Jarvella (1973).

Comparing the semantic retention functions for same vs new conditions with those for paraphrase vs new conditions reveals a very small but consistent difference between the two. The retrieval of semantic information is slightly superior for same-structure sentences than for paraphrases at both low and high degrees of memorability. The slight superiority of recognition memory for same sentences over paraphrases could indicate some interaction with syntactic-lexical structure in retrieval from semantic memory. Alternatively, the paraphrases may simply have been slightly less than perfect, and this was reflected in slightly inferior memory performance. Also, if the paraphrases imperfectly preserved meaning, some proportion of the paraphrases might have been detected as being different from the original sentences in wording because of slight differences in meaning. 


\section{REFERENCES}

Begg, I. Recognition memory for sentence meaning and wording. Journal of Verbal Learning \& Verbal Behavior, 1971, 10, 176-181.

Begg, I., \& Paivio, A. Concreteness and imagery in sentence meaning. Journal of Verbal Learning \& Verbal Behavior, $1969,8,821-827$

Begg, I., \& Robertson, R. Imagery and long-term retention. Journal of Verbal Learning \& Verbal Behavior, in press.

Bransford, J. D., Barclay, J. R., \& Franks, J. J. Sentence memory: A constructive versus interpretive approach. Cognitive Psychology, 1972, 3, 193-209.

Bransford, J. D., \& Franks, J. J. The abstraction of linguistic ideas. Cognitive Psychology, 1971, 2, 331-350.

Franks, J. J., \& Bransford, J. D. The acquisition of abstract ideas. Journal of Verbal Learning \& Verbal Behavior, 1972, $11,311-315$

Jarvella, R. J. Coreference and short-term memory for discourse. Journal of Experimental Psychology, 1973, 98, 426-428.

Johnson-Laird, P. N., \& Stevenson, R. Memory for syntax. Nature, 1970, 227, 412 .

Sachs, J. S. Recognition memory for syntactic and semantic aspects of connected discourse. Perception \& Psychophysics, $1967 \mathrm{a}, 2,437-442$.

Sachs, J. S. Recognition of semantic, syntactic and lexical changes in sentences. Presented at the meeting of the Psychonomic Society, $1967 \mathrm{~b}$.

Shepard, R. N. Recognition memory for words, sentences, and pictures. Journal of Verbal Learning \& Verbal Behavior, 1967, $6,156-163$

Wanner, H. E. On remembering, forgetting, and understanding sentences: A study of the deep structure hypothesis. Unpublished doctoral dissertation, Harvard University, Cambridge, Massachusetts, 1968.

Wickelgren, W. A. Trace resistance and the decay of long-term memory. Journal of Mathematical Psychology, 1972a, 9, 418455 .

Wickelgren, W. A. Strength-resistance theory of the dynamics of memory storage. In D. H. Krantz, R. C. Atkinson, R. D. Luce, and P. Suppes (Eds.), Contemporary developments in mathematical psychology. San Francisco: Freeman, 1972b.

(Received for publication August 13, 1973; revision received October $17,1973$. ) 